\title{
LES ECHANGES ERASMUS EN SUISSE
}

\author{
Soledad Perez" \\ Claudio Bolzman"
}

\section{Introduction}

Le 5 juin 2002, à l'Université de Genève, les échanges Erasmus ont fêté leurs dix ans comme dans toute l'Europe. Des étudiants et des enseignants de trois hautes écoles genevoises (Université de Genève, Institut d'études sociales (IES), Ecole supérieure des arts visuels ont pu faire-part de leurs pratiques et observations dans le cadre des échanges européens. Outre l'aspect festif et ludique, cette journée a été également l'occasion de faire le point sur la participation de la Suisse. Il faut rappeler que la Suisse n'est plus membre d'Erasmus depuis 1995. Faisant suite au refus par votation populaire (le 6 décembre 1992) de l'adhésion à l'Espace Economique Européen (EEE), l'accord officiel entre la Suisse et l'Europe est devenu caduc trois ans après ; Pour trouver une place dans ces échanges, la Suisse a négocié un accord transitoire que nous appelons " association silencieuse".

Dans une première partie, nous définirons le concept d'échanges lié à deux autres concepts qui sont l'altérité et l'équité. Le croisement de ces trois concepts apporte, selon nous, des dimensions intéressantes quant aux contexte socio-économique et éducatif des participants à ces échanges Erasmus.

En deuxième partie, nous étudierons la situation des échanges Erasmus en Suisse et plus particulièrement à l'Université de Genève et à la future Haute Ecole spécialisée Sociale-Santé de la Suisse occidentale (HESS2), deux institutions tertiaires suisses, l'une à tendance académique et l'autre à tendance professionnelle même si les deux organisations développent les deux dimensions. Nous avons choisi la Section des Sciences de l'Education (SSED) de la Faculté de Psychologie et des Sciences de l'Education de l'Université de Genève et l'Institut d'Etudes Sociales (IES) de la Haute Ecole spécialisée SocialeSanté de la Suisse occidentale (HESS2 qui participent aux échanges Erasmus.

En troisième partie, nous nous intéresserons aux statistiques pour comprendre les flux d'étudiants suisses partant pour l'étranger et d'étudiants européens venant à Genève.

En quatrième partie, nous nous demanderons quelles sont les incidences du processus de Bologne sur les échanges Erasmus

Enfin, nous conclurons notre contribution par une perspective de recherche.

\footnotetext{
* Université de Genève.

** Université de Genève et Institut d'Etudes Sociales (IES)
} 


\section{Les échanges Erasmus \\ - Définition des Echanges}

Le concept d'échanges est développé en Education comparée et représente un des concepts-clés de cette discipline. Plusieurs auteurs ont défini ce concept en l'associant au voyage. Par exemple, La Borderie (2000:9) a parlé du paradoxe du voyageur en insistant sur la mobilité : "En voyage, on $v a$, on vient, on observe, on fait un rapport et le temps d'écrire le rapport, les choses ont déjà changé ". Les compétences acquises lors d'un voyage d'agrément et/ou d'études permettent de mieux comprendre les autres et d'échanger. Calderon (2000 :141) rappelle que " dans la mobilité, il existe plusieurs niveaux d'échanges dont ceux qui ont lieu sur le lieu d'accueil, le plus souvent bien ancrés sur des faits pratiques en ce sens que savoir " voyager ", c'est recréer les éléments de la vie quotidienne, acquérir une compétence qui permet d'échanger avec les autres mais aussi tous ceux qui prolongent le séjour à l'étranger. "

Selon notre expérience de responsables Erasmus, les objectifs des échanges dans les pays européens (y compris la Suisse) sont de favoriser la découverte d'autres cultures, l'ouverture à des réalités différentes, l'apprentissage d'autres langues, la capacité de se décentrer; d'expérimenter l'immersion dans un autre contexte socioculturel, de développer des compétences pour se débrouiller dans une réalité connue à divers niveaux; d'encourager la découverte d'autres modes de concevoir et de pratiquer les expériences acquises dans le panorama d'étudiant-e ; de favoriser les échanges entre étudiants et enseignants dans l'optique d'accroître des contacts humains par delà les frontières, mais également de créer des réseaux de professionnels avec une vision plus internationale et comparative. On le constate ici les échanges sont indéniablement liés à l'altérité comme le fait remarquer Kohler-Bally (2001).

\section{- Echanges et altérité}

Les échanges Erasmus amènent les étudiants et les enseignants du tertiaire à la décentration et à la vision de l'altérité. Perez, Groux et Ferrer (2001) ont relevé que les problématiques, étudiées sous des angles différents, permettent une décentration de l'individu, une prise de recul et un changement de point de vue qui favorisent l'analyse et facilitent la compréhension. En effet, c'est par la rencontre de l'autre, dans son pays, que l'on pourra prendre conscience des différences dans tous les domaines de la vie sociale, politique, économique, culturelle, mais aussi dans le domaine éducatif. L'accès au savoir relatif aux systèmes éducatifs est donc facile et chacun d'entre nous peut recourir à des comparaisons internationales pour mieux comprendre ce qui se passe dans les autres pays, mais aussi dans son propre système éducatif. De la 
rencontre avec l'autre, avec son institution éducative, son histoire, sa culture, naît un indéniable enrichissement, que l'on soit élève, étudiant, enseignant, chercheur ou responsable éducatif. Par cette rencontre, on peut opérer des rectifications de détail ou des modifications plus profondes: sur le plan pédagogique (reconsidérer par exemple le problème du redoublement, de l'apprentissage précoce des langues...) ou sur le plan culturel (confronter ses normes et ses valeurs à celle des autres, comprendre les différences).

Comprendre l'autre c'est aussi faire preuve de tolérance envers l'autre. Les organisations internationales et les Etats ont proposé des politiques éducatives qui intègrent des cours d'éducation à la citoyenneté, d'éducation à la paix, d'éducation civique dans les sous-systèmes éducatifs (Perez, 2002). A ce sujet, un des guides éducatifs des Nations Unies est un exemple intéressant. Un texte à l'intention des enseignants relève les objectifs (1995:7): "L'éducation internationale doit viser la survie de l'humanité ; elle doit promouvoir un combat collectif contre l'inégalité, l'injustice et la violence. "Le 6e thème (1995 : 28-29) aborde la "Tolérance, le nouveau nom de la paix ". Dans ces deux pages, le guide propose une fiche d'information sur l'Année internationale pour la Tolérance et la définition de la persécution : " c’est lorsque vous n'avez pas le droit : de pratiquer la religion de votre choix, de parler votre langue, d'exprimer vos idées, de suivre vos opinions politiques, de vous déplacer dans votre pays, de sortir de votre pays, de publier vos idées. Vous perdez votre liberté. ". Il propose également des travaux pratiques sur la définition de la tolérance, sur les droits des jeunes. Non seulement les curriculum doivent intégrer des cours d'éducation internationale mais également encourager les étudiants à faire des expériences dans d'autres systèmes tertiaires. Or, les échanges Erasmus représentent certains inconvénients en ce qui concerne l'équité.

\section{- Echanges Erasmus et équité: des difficultés économiques et administratives; des avantages sociaux}

Nous allons nous intéresser à quelques difficultés qui freinent à notre sens les échanges Erasmus.

La première difficulté est d'ordre économique : Nous identifions dans cette partie les obstacles rencontrés par les jeunes issus des classes populaires et les facilités de partir pour les étudiants des classes moyennes et supérieures en raison de l'appui de leurs parents. En Suisse, mais également en Europe, les problèmes financiers dépendent étroitement de la modestie des bourses (le Bureau Erasmus suisse accorde entre CHF 220,- et CHF 260,- par mois pour les étudiants sortants ; entre CHF 220,- et CHF 280,- - pour les étudiants arrivants $^{1}$ ). Seuls ceux qui ont des ressources ou des relations dans le pays d'arrivée partent. Il est intéressant de constater que cette contrainte économique 
a été signalée par l'Union européenne. Le rapport de la Commission "Enquête sur la situation socio-économique des étudiants Erasmus " signale qu'il serait bon " d'envisager d'autres formes de soutien indirect (réductions tarifaires sur les voyages, aides au logement, systèmes de prêt destinés à couvrir les coûts de la mobilité, etc.) qui viendraient s'ajouter à la bourse d'Erasmus pour en renforcer l'efficacité. "Heaton-Harris (2000:6). On peut également constater que les étudiants-e-s avec des charges de famille, ou un handicap physique ou bien encore dépendant d'un revenu annexe n'ont guère de chances de participer à la mobilité estudiantine.

La deuxième difficulté est d'ordre institutionnel : il est compliqué de faire reconnaître comme équivalente la partie de la formation faite à l'étranger. Les exigences cumulées de l'école de départ et de celle d'accueil sont parfois divergentes et il est constaté que les deux institutions gèrent cette difficulté de manière ponctuelle, selon les étudiants et la bonne volonté des enseignants.

La troisième concerne la réinsertion dans l'université d'origine car cette expérience d'échanges est souvent bénéficiaire au niveau personnel mais il existe un manque d'échanger ces expériences nouvelles avec d'autres jeunes au retour de l'échange dans divers contextes.

La quatrième est de nature juridique : les accords de Schengen introduisent une logique binaire : libre circulation pour les membres de l'UE, forte limitation à l'entrée sur le territoire européen pour les " extra-européens ". Les limites de cette logique binaire apparaissent dans le cas des résidents extra-européens. Ils résident dans un Etat, mais ont besoin d'une autorisation voire d'un visa pour se déplacer (Bolzman, 1993); c'est également le cas s'ils souhaitent participer aux échanges Erasmus avec la Suisse. Par ailleurs, la Confédération introduit ses propres limitations. Ainsi, par exemple, une étudiante brésilienne mariée depuis moins d'un an avec un ressortissant suisse, risque de perdre son permis de séjour si elle part pour six mois avec Erasmus en France. Un requérant d'asile étudiant n'a pas le droit de quitter la Suisse.

La cinquième relève de l'administration: pour les étudiants étrangers, la complexité de l'assurance maladie suisse et les exigences administratives constituent parfois un instrument de blocage à la mobilité. Le logement est une difficulté supplémentaire surtout pour les étudiants Erasmus venant à Genève où la pénurie d'appartements et/ou de chambres est une constante ces dernières années. Pour les enseignants, la lourdeur administrative les freine et ils hésitent à s'engager dans des échanges privilégiant plutôt la recherche qui leur permettra de publier des ouvrages et des articles scientifiques indispensables pour la carrière académique.

Pourtant, il existe indéniablement des avantages que nous avons identifiés ci-après. 
Les avantages sociaux humains nous paraissent très importants. Nous avons vu que les échanges améliorent les représentations sociales et individuelles. Ils permettent d'acquérir des compétences sociales et humaines sans oublier des compétences linguistiques. Au niveau individuel, la personne peut échanger avec d'autres de diverses cultures et comparer la situation de l'ailleurs à son propre contexte. Nous espérons que ces compétences sociales et humaines (respect, tolérance, ouverture à la diversité culturelle entre autres) restent acquises définitivement.

Les avantages sociaux professionnels concernent les relations professionnelles entre enseignants notamment.

Le réseau européen des écoles et universités est un exemple qui permet une meilleure connaissance de ce qui se fait dans d'autres pays. En ce qui concerne les échanges Erasmus étudiants, nous postulons que les échanges se font grâce aux réseaux des enseignants des deux institutions. Nous relevons ce fait car nous sommes persuadés que les échanges Erasmus n'existeraient pas sans le volontariat et la mise à disposition de réseaux par les enseignants. Certains sont intéressés par la possibilité d'échanges enseignants avec des collègues de diverses cultures en ce qui concerne l'enseignement, la recherche et les modes d'intervention dans la société civile. Mais regardons de plus près la situation Erasmus en Suisse et plus particulièrement à Genève, ville internationale.

\section{Les échanges Erasmus actuels}

\section{En Suisse}

Comme nous l'avons souligné en introduction c'est à partir de 1995 que la Suisse finance les échanges avec ses partenaires. Ce statut particulier n'a pas empêché l'augmentation de la participation financière suisse lors des dernières quatre années.

En effet, la participation est passée de 5.26 millions de francs en 1997/98 pour soutenir les activités de mobilité de 23 Hautes écoles, à 7.97 millions de francs pour encourager les activités de 37 hautes écoles en 2002/ 03. L'essentiel de cette somme ( 5.75 millions) est consacré aux bourses de mobilité, 1.83 millions sont utilisés pour les autres activités de mobilité des hautes écoles (échanges d'enseignants, visites préparatoires, ECTS, etc.) et 0.39 millions pour des mesures d'accompagnement visant à faciliter la mise en place de la mobilité dans les hautes écoles non universitaires (OFES, 2002).

En 2001-2002, 1353 étudiant-e-s ont quitté la Suisse vers une autre université ou haute école européenne et 1584 étudiant-e-s sont venus en Suisse. Les destinations les plus prisées des étudiants suisses en 2001-2002 
sont l'Allemagne (313 étudiants), l'Allemagne (229), la Allemagne (205) la Grande Bretagne (169) et l'Allemagne (102). Quant aux étudiants européens qui choisissent la Suisse comme destination, ils proviennent principalement de ces cinq pays : l'Allemagne (472), l'Allemagne (219), la Allemagne (170), la Grande Bretagne (120) et l'Allemagne (120). On observe les mêmes tendances en 2000-2001 (Bureau Erasmus, 2002a).

Au niveau des Universités suisses, en 2000-2001, la mobilité a concerné 177 étudiants de l'Université de Berne, 156 de l'Université de Fribourg, 148 de l'Université de Zurich, 144 de l'Université de Genève et 134 de l'Université de Lausanne. En 2001-2002, ces chiffres ont varié légèrement (Bureau Erasmus, 2002a).

En ce qui concerne l'Université de Genève, les destinations préférées sont assez proches de celles observées au niveau Suisse : Allemagne, Grande Bretagne, par exemple. Ce sont les étudiants de l'Ecole de traduction et d'interprétation qui se déplacent le plus à l'étranger. Les filles s'intéressent plus que les garçons à la mobilité estudiantine. En 2000-01, 133 d'entre elles ont pris part au programme contre 37 hommes (Bureau Erasmus, 2002b).

Il existe bien évidemment plusieurs types d'échanges et d'accords bilatéraux avec des universités étrangères européennes et du sud mais nous allons nous intéresser uniquement aux échanges Erasmus en Sciences de l'Education (SSED) et à l'Institut des Etudes Sociales (IES). Tout d'abord, nous décrirons succinctement l'historique des échanges. Pour ce faire, nous partirons de l'exemple de l'IES que nous comparerons à celui de SSED.

\section{Historique des échanges à l'IES et en Sciences de l'Education}

\section{- L'Institut d'études sociales}

L'Institut d'études sociales (IES) a été fondé en 1918. L'IES comprend, outre l'Ecole supérieure de travail social (ESTS), l'Ecole de psychomotricité, le Centre de formation continue (CEFOC) et le Centre de recherche sociale (CERES). Les principales activités de l'Institut sont :

- la formation de niveau tertiaire professionnel pour les travailleurs sociaux et psychomotriciens ;

- la formation continue de travailleurs sociaux, y compris dans le domaine de l'aide à domicile, de la petite enfance et des établissements médico-sociaux. Plusieurs certificats post-grade sont proposés : par exemple, intervention systémique, intervention collective, médiation familiale, etc.

- la recherche dans le domaine du travail social et de la 
psychomotricité; les projets menés à l'IES sont financés par le FNRS (programmes nationaux de recherche), des mandats des collectivités publiques, les programmes européens, les ressources internes ;

- la publication des ouvrages dans le domaine social par le biais des Editions IES ;

- la production de films dans le domaine social.

\section{- Les Sciences de l'Education}

L'histoire des Sciences de l'Éducation à l'Université de Genève est intimement liée à l'histoire de la psychologie. En 1912, l'Institut Jean-Jacques Rousseau est créé par Th. Flournoy (nommé professeur de psychologie à la Faculté des Sciences) et E. Claparède. Cet institut a comme objectifs : une école pour éducateurs, un centre de recherche sur le développement de l'enfant et les techniques d'apprentissage, un centre d'information et un centre de diffusion des thèmes de l'éducation nouvelle et de l'école active. Au départ, l'institut est une fondation privée qui sera rattachée à l'Université en 1948. En 1921, la nomination de J. Piaget apportera une dimension particulière. En 1929, l'Institut est rattaché à la faculté des Lettres. J. Piaget devient le directeur du Bureau International d'Education de 1929 à 1968. En 1933, la formation des enseignants primaires comporte une année à l'institut. En 1955, J. Piaget crée le centre international d'épistémologie génétique. En 1970, l'Institut devient l'école de Psychologie et des Sciences de l'Education. En 1975, l'école devient la septième faculté de l'Université de Genève.

\section{Les études dans les deux institutions}

\section{- La formation en travail social à l'école supérieure de travail social (ESTS)}

L 'ESTS offre une formation au travail social dans trois filières professionnelles :

l'animation socioculturelle, l'éducation spécialisée et le service social. D'une durée normale de trois ans, cette formation débouche sur un Diplôme en travail social, avec indication de l'option professionnelle.

L'ESTS accueille chaque année environ 60 étudiants dans la formation plein temps, 20 étudiants dans la formation à temps partiel, et 35 dans la formation en emploi. Les formations à temps partiel et en emploi ont une durée d'environ quatre ans. En tout, un peu plus de 300 étudiants suivent simultanément la formation en travail social. C'est donc une école de dimension 
modeste qui fonde sa démarche pédagogique sur l'enseignement à des groupes restreints, et sur l'accompagnement individuel et collectif des étudiants tout au long de leur formation.

A partir de l'année académique 2002-2003, l'IES et ses quatre filières de formation professionnelle feront partie de la Haute école spécialisée socialesanté de la Suisse occidentale (HESS2). Il deviendra un des quatre sites de la Suisse romande (avec les écoles de Fribourg, Lausanne et Sion) où une haute formation spécialisée en travail social sera dispensée. La création des HES vise à améliorer la qualité de la formation professionnelle de niveau tertiaire en Suisse, ainsi qu'à faciliter la reconnaissance des diplômes au niveau suisse et européen.

\section{- Les études en SSED}

La Section des Sciences de l'Education propose actuellement une licence (240 crédits) avec trois mentions (mention enseignement, mention recherche et intervention, mention, formateur d'adultes). Le troisième cycle relativement récent se compose d'un DEA, d'un DESS et d'un doctorat. Le DEA (60 crédits) a 3 options dont l'option Didactiques, l'option Processus d'apprentissage et d'enseignement en contexte et l'option Sociétés et systèmes d'éducation. En 2002, les futures transformations du système universitaire avec le processus de Bologne affecteront les plans d'études de la Section.

SSED accueille 1177 étudiants au total (les 3 cycles confondus) en 2001-2002, environ la moitié des étudiants de la Faculté avec 779 étudiants au $2^{\text {e }}$ cycle, 259 en $1^{\text {er }}$ cycle et 139 en $3^{\text {e }}$ cycle.

Néanmoins, signalons que des enseignants de SSED organisent des semaines d'études pour étudiants et/ou enseignants à l'étranger ce qui permet de mieux se connaître et d'interagir avec des collègues étrangers.

\section{La place de la coopération internationale à l'IES et en SSED}

\section{- A l'IES}

L'IES a depuis près de 50 ans des contacts et des échanges avec des écoles de travail social de l'étranger (Weber, 1999). Il est membre de trois associations d'écoles européennes : L'Association européenne des écoles de service social (EASSW) ; l'Association européenne des centres de formation au travail socio-éducatif (FESET) ; le Centre européen d'éducation communautaire (ECCE).

L'IES, et en son sein l'Ecole supérieure de travail social, s'intéresse dès le début des années 1990 à la Coopération européenne. Durant l'année 
académique 1990-1991, un Groupe "Europe " voit le jour dans le but de développer la participation de l'IES aux réseaux européens existants et de promouvoir la coopération avec certaines écoles partenaires. Il s'agit en particulier d'organiser des visites préparatoires, de mettre sur pied des séminaires intensifs communs, de favoriser les échanges d'étudiants suivant la formation à plein temps (trop compliqué pour les FEE) et d'enseignants.

Avec des prémisses prometteuses dans les années 90-93, c'est lors de l'année 1993-1994 que les activités de mobilité se développent. L'IES coordonne un PIC et participe à deux autres. C'est à l'intérieur de ces PIC que s'organisent les échanges d'étudiants et que des séminaires intensifs d'une dizaine de jours sur divers thèmes sont proposés. Par ailleurs, une semaine de mobilité est instaurée durant le mois de février pour favoriser la tenue des séminaires intensifs, mais également pour permettre à des étudiants de découvrir des écoles ou institutions dans d'autres pays ou en Suisse. Un fonds est créé pour encourager les départs des étudiants pendant cette semaine : chacun reçoit CHF 100,- - Cette fenêtre de mobilité est destinée aux étudiants de deuxième année à plein temps : 25 étudiants sur 75 s'inscrivent pour participer à cette expérience. Les destinations retenues pour la première année sont Valencia, Grenoble, Turin, Berne, Paris, et un groupe itinérant Morges, Tavannes, Bâle. Les étudiants reçoivent des crédits de formation pour cette activité. Les activités de coopération européenne s'institutionnalisent, que ce soit en termes d'échanges d'étudiants, de participation à des séminaires intensifs, de la semaine de mobilité. Chaque année, 4-5 étudiants en moyenne vont à l'étranger et l'IES reçoit un nombre équivalent d'étudiants. Le seul objectif que le groupe n'arrive pas à institutionnaliser dans le court terme est la mise en place d'un cours sur le travail social en Europe.

Quant à la structure chargée de gérer l'ensemble des échanges, elle est modeste. Le Groupe est composé du directeur de l'IES, du directeur de l'ESTS, de trois enseignants et d'une secrétaire à temps très partiel. Ils se réunissent une fois tous les deux mois, chacun ayant des tâches spécifiques à assumer.

\section{- Dans la Section des Sciences de l'Education}

Les Echanges Erasmus occupent peu de place tant au niveau des étudiants que des enseignants. Ces échanges avec l'Europe ont lieu en raison bien souvent des relations professionnelles des enseignants de la Section avec des collègues européens. Ensuite, certains d'entre eux font des expertises internationales qui agrandissent les réseaux des connaissances professionnelles. On l'a vu dans les statistiques, le plus grand nombre d'étudiants SSED se trouvent au $2^{\mathrm{e}}$ cycle, période favorable pour les échanges qui sont peu 
encouragés par les enseignants. La structure est composée des enseignants qui s'occupent de la partie académique et la conseillère aux études qui gère la partie administrative.

\section{Les moyens à disposition dans les deux institutions}

Une " fenêtre de mobilité " pour les deux institutions qui gèrent les dossiers Erasmus de manière différente: La conseillère aux études + l'enseignant responsable de l'échange pour SSED qui approuve les échanges par le Collège des Professeurs de la Section. L'IES gère les dossiers Erasmus comme il a été déjà mentionné plus haut.

Les étudiants sont encouragés à partir pendant le $3^{\text {ème }}$ semestre de la formation à l'IES, pendant le $2^{\text {nd }}$ cycle en SSED. Ils peuvent prendre à la fois un stage et des cours dans une autre université européenne.

L'IES a un éventail d'accords bilatéraux avec 21 universités et écoles européennes avec lesquelles des contacts réguliers sont entretenus.

L'Université de Genève a conclu des accords avec des institutions étrangères dont les échanges Erasmus qui représentent pour la faculté, environ 49 accords dont 23 au niveau facultaire (possibilité pour tous les étudiants de la faculté), 5 accords des Sciences de l'éducation et 21 pour la psychologie.

Une information personnalisée est proposée aux étudiants par un département des relations internationales à l'Université de Genève et à l'IES par l'équipe compétente. Une aide administrative et psychosociologique à la préparation du départ par les services responsables des échanges est organisée pour aider les étudiants à préparer leur voyage d'études.

Le système ECTS des stages et cours pris à l'étranger est validé dans les deux institutions. L'IES joue le jeu Erasmus et délègue aux universités partenaires l'évaluation des actes de formation réalisés à l'étranger, ce qui n'est pas le cas de toutes les universités.

L’Université de Genève laisse la possibilité de l'évaluation dans le pays d'origine ou dans le pays d'accueil.

Un accompagnement minimal lors du retour des étudiants pour favoriser la prise de parole et la réintégration est proposé à l'IES. Cette fonction est remplie par une conseillère aux études en Sciences de l'Education.

Nous venons d'analyser les structures. Il convient maintenant de regarder de plus près les statistiques. 


\section{Sta tistiques}

Si la conception des échanges Erasmus est intéressante ainsi que son déroulement, les statistiques restent importantes pour comprendre la place des échanges Erasmus dans les deux institutions.

Tableau - Nombre d'étudiants qui partent et qui rentrent par année académique

\begin{tabular}{|c|c|c|c|c|c|c|c|c|}
\hline Année & In & IES & SSED & Out & IES & SSED & Total IES & SSED \\
\hline 1996-97 & & 8 & 9 & & 9 & 2 & 17 & 11 \\
\hline $1997-98$ & & 8 & 14 & & 9 & 2 & 17 & 16 \\
\hline $1998-99$ & & 9 & 8 & & 3 & 2 & 12 & 10 \\
\hline $1999-00$ & & 6 & 4 & & 7 & 1 & 13 & 5 \\
\hline 2000-01 & & 6 & 1 & & 3 & 5 & 09 & 6 \\
\hline 2001-02 & & 6 & 5 & & 6 & 2 & 12 & 7 \\
\hline Total & & 43 & 41 & & 37 & 14 & 80 & 55 \\
\hline
\end{tabular}

Sources : statistiques de l'IES, SSED (1996-2002).

\section{Destinations}

\section{IES}

Le nombre d'étudiants qui partent dans le cadre des échanges Erasmus est presque identique à celui des étudiants rentrants. Cependant, on observe une diminution des étudiants sortants à partir de1998-99. La raison principale est liée au fait qu'à partir de l'année académique 1998-1999, l'IES a signé un accord bilatéral avec les universités du Québec qui permet aux étudiants de l'Institut de faire gratuitement entre un semestre et une année dans une université québécoise. Huit étudiants sont partis pour le Québec en 1998-1999, ce qui fait un total de 11 étudiants mobiles cette année là. En moyenne, si l'on comptabilise les départs en Europe et au Québec, 9 étudiants d'une volée font une partie de leur formation à l'étranger. En général, une volée plein temps de deuxième année compte environ 70 étudiants, cela veut dire que près de 13\% des étudiants d'une volée font une partie de leur formation à l'étranger, ce qui est considérable. Si l'on considère uniquement les départs vers l'Europe, 8.8\% de la volée. 


\section{SSED}

Il est important de constater que les étudiants de l'Institut partent plus facilement que ceux de la Section des Sciences de l'Education. Un petit nombre d'étudiants se déplacent un semestre ou une année à l'étranger pendant les études universitaires. Il s'avère que les enseignants donnent peu d'informations voire aucune quant aux possibilités européennes tandis qu'à l'IES, l'information est très diffusée. Autre inconvénient majeur, les cours en $2^{\text {ème }}$ cycle sont annuels et non semestriels ce qui crée des complications d'équivalences entre les universités.

L'Université de Genève a également des accords avec les autres universités suisses mais le nombre d'étudiants se déplaçant dans un autre canton reste très faible en Sciences de l'Education comme il est mentionné ciaprès.

Tableau - nombre d'étudiants par pays de provenance et de destination de 1996 à 2001

\begin{tabular}{|c|c|c|c|c|c|c|}
\hline Pays & Et. In & SED & Et. Out IES $\mathbf{S}$ & SSED & Total ES & SSED \\
\hline Allemagne & & 11 & & 03 & & 14 \\
\hline Belgique & 07 & 03 & 05 & 01 & 12 & 04 \\
\hline Danemark & \multicolumn{2}{|c|}{01} & & & & \\
\hline Espagne & 11 & 03 & 13 & 05 & 24 & 08 \\
\hline France & \multicolumn{2}{|c|}{03} & 12 & 04 & 15 & 04 \\
\hline $\begin{array}{l}\text { Grande } \\
\text { Bretagne }\end{array}$ & \multicolumn{2}{|r|}{29} & 02 & 04 & 02 & 33 \\
\hline Irlande & \multicolumn{2}{|r|}{--} & \multicolumn{2}{|r|}{04} & \multicolumn{2}{|r|}{04} \\
\hline Italie & 15 & 02 & \multicolumn{2}{|c|}{05} & \multicolumn{2}{|r|}{02} \\
\hline Suède & \multicolumn{2}{|r|}{--} & \multicolumn{2}{|r|}{01} & \multicolumn{2}{|r|}{01} \\
\hline $\begin{array}{l}\text { Suisse } \\
\text { alémanique }\end{array}$ & \multicolumn{2}{|c|}{$03 \quad 03$} & \multicolumn{2}{|c|}{01} & \multicolumn{2}{|r|}{03} \\
\hline Québec & 03 & 02 & \multicolumn{2}{|c|}{14} & 17 & 02 \\
\hline Amérique du Sud & \multicolumn{2}{|c|}{--} & \multicolumn{2}{|c|}{05} & \multicolumn{2}{|r|}{05} \\
\hline Afrique & \multicolumn{2}{|r|}{--} & \multicolumn{2}{|r|}{02} & \multicolumn{2}{|r|}{02} \\
\hline Asie & \multicolumn{2}{|l|}{--} & \multicolumn{2}{|r|}{02} & \multicolumn{2}{|r|}{02} \\
\hline
\end{tabular}

Source : statistiques des deux institutions de 1996-2001 


\section{IES}

Quant aux destinations les plus prisées, il s'agit de la France, l'Espagne et, dans une moindre mesure, de l'Italie, la Belgique et l'Irlande. Le Québec est la destination favorite hors Europe. En ce qui concerne les origines des étudiants arrivants, ils viennent principalement de l'Italie, l'Espagne, l'Allemagne, la Belgique. On constate l'existence d'un bon équilibre dans les échanges avec l'Espagne et la Belgique. Dans les échanges avec l'Italie et l'Allemagne, ce sont les étudiants originaires de ces pays qui sont davantage demandeurs. Par rapport à la France et aux pays anglophones, ce sont les étudiants de Genève qui sont les plus intéressés. On observe le même phénomène dans les échanges avec le Québec.

Parmi les partants, on trouve un nombre important de jeunes issus de la migration qui vont en Espagne et en Italie : sur les 18 départs vers ces deux pays, 14 concernent des jeunes de la deuxième génération. Ces jeunes ont plusieurs atouts qui facilitent leur mobilité : ils connaissant la langue du pays, ont parfois de la parenté qui peut les héberger, ont eu l'opportunité de sa familiariser avec la situation sur place lors des séjours de vacances. Pour ces jeunes, Erasmus est un programme qui permet d'actualiser leur " bilatéralité de références" (Bolzman, Fibbi, Vial, 2002). Cette dimension mériterait des recherches ultérieures, d'autant plus que sur les 27 autres étudiants ayant séjourné en Europe, 5 sont également de la " deuxième génération " . On connaît en effet peu d'informations sur les caractéristiques sociologiques des étudiants mobiles.

\section{- SSED}

Le pays avec lequel la Section des Sciences de l'Education a le plus de contacts est la Grande-Bretagne ce qui s'explique par les liens professionnels et de réseaux d'une enseignante.

Les Sciences de l'Education attirent les étrangers mais les étudiants suisses ou qui vivent en Suisse ne semblent pas beaucoup attirés par les échanges car une des mentions de la licence en Sciences de l'Education, la mention enseignement ne permet en aucun cas une mobilité par la structure même de son cursus et l'importance des stages dans les écoles genevoises. Les deux autres mentions recherche et intervention et formateur d'adultes semblent avoir plus de souplesse pour des échanges. Les étudiants qui partent à l'étranger proviennent de ces deux mentions pour le $2^{\text {nd }}$ cycle. Pour le $3^{\text {ème }}$ cycle, les étudiants des trois mentions peuvent partir pour l'extérieur de Genève, en Suisse ou ailleurs. 


\section{Les échanges Erasmus dans le processus de Bologne}

Il convient de signaler d'ores et déjà que le processus de Bologne représentera de grands changements dans les échanges Erasmus selon les domaines d'études, selon les règlements d'études des nouveaux cursus et le financement des échanges.

Les échanges Erasmus représentent pour les acteurs décideurs une efficacité économique à court terme (professionnalisation) et une découverte et formation à l'altérité jugée nécessaire dans l'Union Européenne.

Au niveau de la structure des études universitaires, la compatibilité des cursus devrait s'améliorer par l'introduction des deux cycles BA/MA ; pourtant, le premier cycle est raccourci à trois ans et semble rendre plus difficile la mobilité (undergraduate) et par conséquent, le programme Erasmus sera prétérité. Par ailleurs, l'augmentation de la sélectivité au $2^{\text {ème }}$ cycle va limiter la mobilité dans les plans d'études.

Pour les diplômes, les contenus des cursus académiques menant au même titre varient fortement et accentuent la difficulté de la mobilité ; l'aspect compétitif de Bologne incite les universités à devenir plus protectionnistes, à garder leurs compétences/skills précieusement ce qui représenterait une limitation pour la mobilité.

L'adoption partout d'un système ECTS facilitera la mobilité inter européenne et on devrait avoir une meilleure mesure des équivalences, le développement de "study levels indicators ", "learning outcomes indicators " facilitera la mobilité.

Selon la typologie développée par le groupe de travail suisse chargé du dossier de la mobilité, quatre types de mobilité semblent devoir prendre de l'importance à l'avenir (Vincent, 2002):

La mobilité programme fenêtre organisée : elle est facultative, à l'intérieur d'un cursus académique ( $\mathrm{Ba}, \mathrm{Ma}, \mathrm{PhD}$ ) et dans le cadre d'un programme d'échange (géré par un bureau administratif en collaboration avec les facultés), lors d'une "fenêtre de mobilité". La fenêtre sert à faciliter l'organisation de la mobilité de type ERASMUS au niveau Ba, Ma ou PhD. C'est une période prévue spécialement dans le règlement d'études pour effectuer un séjour d'études dans un autre établissement universitaire. La fenêtre est mise en place de façon coordonnée avec les autres signataires de la Déclaration de Bologne. La mobilité "fenêtre" est ouverte sur l'Europe comme sur le reste du monde. La mobilité "fenêtre" utilise l'ECTS pour la reconnaissance pleine et entière des séjours à l'étranger (cours, crédits, notes).

La mobilité "fenêtre" n'est pas obligatoire. La mobilité "fenêtre" n'est pas nécessairement organisée dans le cadre d'un programme d'échange. 
La mobilité diplôme multiple : elle est obligatoire, lors d'un cursus académique $(\mathrm{Ba}, \mathrm{Ma}, \mathrm{PhD})$, menant dans certains cas à un "diplôme multiple ou conjoint".

La mobilité "diplôme multiple/conjoint" nécessite des liens forts entre établissements, tant au niveau académique qu'administratif. Il est important de définir le type de diplôme obtenu : multiple, conjoint, certificat, (avec/sans supplément au diplôme ?).

Il faut évaluer les conséquences légales : règlements d'études, reconnaissance, financement des établissements.

Il est impossible d'obliger chaque étudiant à être mobile et d'instaurer ce type de diplôme dans tous les cursus.

La mobilité verticale permet généralement l'immatriculation dans un établissement différent mais il existe des obstacles qui ont été pointés par le groupe de travail suisse dont les Obstacles financiers qui sont importants. Par exemple, les étudiants qui s'exmatriculent du système éducatif suisse ne bénéficient plus dans la majorité des cas, d'une allocation d'études.

Dans le système Ba-Ma, les taxes d'écolage des Ma risquent d'être élevées dans beaucoup de pays européens. Il est connu que les pays anglophones ont des taxes importantes notamment pour les pays non européens comme la Suisse. D'une part, le coût effectif d'un Ma sera fort probablement supérieur au coût de la licence. Dans l'Union européenne, des masters apparaissent dans des établissements tertiaires publics ou privés qui peuvent être très onéreux pour les étudiants. En règle générale, un séjour de mobilité ne devrait pas faire perdre les avantages sociaux dont un étudiant bénéficie dans son pays d'origine.

Les obstacles administratifs ont été relevés par le groupe suisse mandaté par la Conférence des Recteurs des Universités Suisses (CRUS) comme la réalisation de la mobilité hors programme. Les étudiants (partants et hôtes) ne bénéficient plus d'allégements administratifs, tels que : information sur les programmes des cours, les modalités d'inscription, les démarches pour l'obtention d'un visa ou la recherche d'un logement.

Les obstacles académiques risquent également de mettre en péril la mobilité. Les étudiants dont la demande d'admission aura été rejetée entreprendront des démarches de "tourisme académique" afin de pouvoir trouver un établissement qui les acceptera.

Les candidats dont la demande d'admission sera rejetée ne seront porteurs que d'un $\mathrm{Ba}$, qui risque d'être considéré comme un diplôme «au rabais".

La mobilité grise est en réalité hors programme et après une exmatriculation, non-organisée. C'est une mobilité complètement libre mais il est très difficile d'en garder une trace et enfin il existe des risques de problèmes de reconnaissance et de transfert de crédits. 
Le groupe de réflexion insiste sur le fait que la mobilité pourrait disparaître si les nouveaux règlements d'études ne tiennent pas compte dès le départ de la mobilité. Ce type de mobilité nécessite des cursus longs ou des périodes spécialement prévues pour permettre une expérience intéressante pour les étudiants et les enseignants. Ces experts suisses relèvent la nécessité d'adapter l'offre de cours aux nouveaux types de mobilité (p. ex : Ma en gestion pour détenteur de Ba en psychologie). Il note également qu'il faudra tenir compte de la compétitivité croissante entre les universités pour attirer les meilleurs étudiants. Il existe donc un risque pour les universités peu compétitives de se voir reléguées en "deuxième position". Enfin, l'impossibilité pour les programmes attractifs d'accepter tous les étudiants est soulevée.

Grâce à la mobilité verticale, les étudiants pourront assembler des diplômes ( $\mathrm{Ba}$ et $\mathrm{Ma}$ ) dans toute l'Europe. Les possibilités de combinaison de ces modules de diplômes seront donc très nombreux.

Toutefois, la quantité de ces combinaisons de diplômes ne doit en aucun cas occulter la "promotion de la nécessaire dimension européenne dans l'enseignement supérieur, notamment en ce qui concerne l'élaboration de programmes d'études, la coopération entre établissements, les programmes de mobilité et les programmes intégrés d'étude, de formation et de recherche." (CRUS, 2001).

Les liens privilégiés entre universités doivent donc se poursuivre pour permettre une offre plus importante de cours pour les étudiants. Néanmoins, la "marchandisation " des offres universitaires notamment pour les Ma risque d'enlever des possibilités aux jeunes issus des classes sociales modestes. Il convient donc d'être très attentifs à cette difficulté tout en sachant que les universités publiques voient de plus en plus réduire leur budget par les autorités politiques qui exigent qu'elles aient des collaborations avec la société civile. Entendons par-là les partenaires économiques qui risquent de jouer ces prochaines années des rôles non négligeables dans la construction de masters universitaires privés et publics. Il faudra donc proposer le maintien des bourses pour le Ma, même à l'étranger et de minimiser les taxes d'écolage du Ma voire même dispenser les bons éléments ce que préconise l'Union européenne dans un rapport de 2001.

Les partenariats et la mobilité verticale restent possibles et peuvent être parallèles et complémentaires. Le groupe de travail a proposé des mesures importantes pour développer les échanges Erasmus dans les nouveaux cursus d'études par exemple de semestrialiser les cours, d'harmoniser le calendrier académique au niveau européen ce qui devient une nécessité, d'accélérer les procédures et d'alléger les contraintes légales liées aux diplômes multiples/ conjoints, d'utiliser systématiquement le système ECTS, d'introduire le Supplément au Diplôme, de développer le système de bourses pour l'apprentissage des langues, de construire des logements-étudiants, de prendre 
des mesures administratives et politiques pour l'obtention de visas, de la non obligation de l'assurance-maladie ${ }^{2}$.

\section{Conclusion}

Il est évident que les étudiants mobiles représentent une faible proportion des étudiants européens. Or, la société civile et en particulier les acteurs économiques apprécient de plus en plus les compétences sociales et humaines acquises par des jeunes professionnels dans des séjours à l'étranger et notamment dans des institutions tertiaires. Nous relevons comme MurphyLejeune (2000) que l'étudiant(e) confronté(e) à une nouvelle réalité transitoire élabore des représentations sociales et humaines et rompt avec les idées stéréotypées sur l'ailleurs et l'étranger. Les étudiants des deux institutions genevoises et suisses ont envie de connaître un espace tertiaire différent mais les contraintes économiques et socio-familiales rendent difficile ce genre d'approche. Il est important de noter que les jeunes issus des migrations peuvent faire valoir leurs compétences linguistiques et leurs réseaux relationnels et cette valorisation représente pour certains un rééquilibrage socio-identitaire.

Un bon exemple des compétences que l'on peut développer à travers la mobilité et la coopération internationale dans le cadre d'Erasmus est représenté par les séminaires intensifs auxquels participe notamment l'IES grâce aux réseaux professionnels.

Un de ces réseaux organise des séminaires autour du thème de la migration, les relations interculturelles et le racisme. Ses membres proviennent des Université d'East London, de Mayence, de Messina, de Valencia, de la Haute école spécialisée de Berne et de l'Institut d'études sociales de Genève. Outre la réflexion autour des contenus, ces séminaires ont permis de réfléchir aux modes de communication lors des échanges internationaux et de développer une méthodologie pour le développement de compétences interculturelles. En Sciences de l'Education, il existe également ces séminaires internationaux qui sont développés non seulement avec les pays du Nord mais également avec les pays du Sud.

Un constat que l'on peut faire est que dans le cadre de séminaires internationaux, on cherche souvent à utiliser une langue commune, en général l'anglais. Ce choix a des avantages, en particulier lorsque l'on dispose de peu de temps pour se rencontrer. Il entraîne également des problèmes qui ne sont pas à négliger : exclusion des personnes qui ne maîtrisent pas bien la langue dominante, construction d'une hiérarchie qui favorise ceux dont la langue maternelle est la langue dominante. La priorité est donnée à un modèle d'analyse et de structuration du monde lié à la langue dominante, une mise en avant de l'efficacité au détriment de la diversité, alors même que le déni de la diversité 
peut faire émerger par la suite des conflits et des problèmes qui sont aussi coûteux en termes de temps et de cohésion du groupe (Kühne, 1999).

Il s'agit donc de chercher un mode de communication qui favorise la participation de tous, tout en tenant compte de la diversité. Ainsi, chacun a le droit de comprendre et d'être compris ; il faut donc un temps pour la clarification. Il est important de souligner que le manque de compréhension ne peut être imputé à la personne qui a des difficultés en raison de ses faibles compétences linguistiques ; la compréhension est un processus relationnel qui concerne les différents partenaires et le groupe dans son ensemble (Kühne, 1997 ; Eckmann, 1998). Il s'agit d'un équilibre qui ne peut pas être donné une fois pour toutes, mais que chaque groupe doit arriver à se construire en fonction de son contexte et de ses interactions concrètes.

Ces apprentissages peuvent par la suite être introduits dans le cadre du programme d'études régulier, en prenant davantage en compte les besoins spécifiques des étudiants issus des minorités (Eckmann, 1998, Bolzman et al 2002).

Une des perspectives de recherche qui nous paraît intéressante est donc de mener une étude sur les compétences humaines et sociales acquises après le séjour à l'étranger en déterminant les étudiants de $1^{\text {ère }}$ génération et de $2^{\text {ème }}$ et $3^{\text {ème }}$ générations pour comprendre les différences et les similitudes de ces publics de jeunes quant aux apports interculturels et aux difficultés rencontrées.

Finalement, nous pouvons constater dans cette analyse comparée entre les deux institutions que d'une part, les échanges Erasmus dépendent d'une volonté politique de soutenir les échanges. Or, force est de constater qu'en Sciences de l'Education, les échanges Erasmus sont peu encouragés et intéressent que rarement des enseignants qui doivent gérer ces dossiers étudiants d'un point de vue académique en plus de leur charge de travail même si la partie administrative est centralisée. D'autre part, les statistiques démontrent que l'IES a une politique beaucoup plus claire que la section des Sciences de l'Education de l'Université de Genève. A l'heure où se discute le processus de la Déclaration de Bologne dans toutes les institutions tertiaires, il sera important de suivre ce dossier qui présente comme nous l'avons vu des impératifs administratifs et académiques importants.

\section{Notes}

1 Ces sommes peuvent être augmentées éventuellement de CHF 50 par mois pour les étudiants. 1 Euro = CHF 1,50 .

2 Le lecteur est invité à consulter le site de l'Université de Genève et son département des relations internationales où sont développés différents points concernant la Déclaration et le processus de Bologne. 


\section{Bbliographie}

Bolzman, C. 1993. Construction européenne, assimilationnisme et citoyenneté. Réflexions sur la place des étrangers résidant dans l'espace européen. In Caloz-Tschopp, M.C. et al. (Ed.), Europe : montrezpatte blanche !, Publicetim, Genève, 385-394.

Bolzman, C., Fibbi, R. \& Vial, M. 2002. "Secondos ". Le processus d'intégration des jeunes issus de la migration en Suisse. Zurich : Ed. Seismo.

Bureau Erasmus Suisse. 2002a. Erasmus-Programm in der Schweiz. Berne. 3.4.02.

Bureau Erasmus Suisse. 2002b. Journée d'action du 5.6.02. Berne.

Calderon, C. 2000. Formation aux échanges. Pratiques d'échanges. In Groux, D. \& Tutiaux-Guillon N. (Eds). Les échanges internationaux et la comparaison en éducation. Pratiques et enjeux. Paris : L'Harmattan.

Conférence des Recteurs des Universités Suisses (CRUS). 2001-2002. Rapport intermédiaire "Déclaration de Bologne ". www.crus.ch/docs/

Eckmann, M. 1998. Instensivseminaire als Erlebnisprozess zu Identität und Mindereheiten in Europa. In Hamburger, F. (Ed.), Faszination und Realität des Interkulturellen, J.Gütenberg Universität Mainz.

Eckmann, M., Bolzman,C. 2001-2002. Quelle place pur l'interculturel dans la formation des travailleurs sociaux? In Ecarts d'identité. Grenoble : n.98. 37-41.

Heaton-Harris. 2000. Enquête sur la situation socio-économique des étudiants Erasmus. Bruxelles : Commission européenne.

Kohler-Bally, P. 2001. Mobilité et plurilinguisme. Fribourg : Editions universitaires Fribourg.

Kühne, K. 1997. I seminari intensivi : una forma di insegnamento per raggiungere la competenza culturale? In Bolognari, V. \& Kühne, K. (Eds.). Povertà, migrazione, razzismo. Bergamo : edizioni junior, 175-192.

Kühne, K. 1999. Communication in international exchanges. In Chytil, O \& Seibel F. (Eds) European Dimensions in Training and Practice of Social professions. Boskovice: Verlag ALBERT, 214-216.

IES. 2002. Programme d'études. Genève : IES.

IES. 2002. Statistiques 1996-2002. Genève : IES. 
La Borderie, R. 2000. L'art du voyage : repères pour la comparaison. In Groux, D. \& Tutiaux-Guillon N. Les échanges internationaux et la comparaison en éducation. Pratiques et enjeux. Paris : L'Harmattan.

Murphy-Lejeune, E. 2000. Mobilité internationale et adaptation interculturelle : les étudiants voyageurs européens. Nancy : INRP.

Nägeli, R., Bologna-Reform und Mobilität, Conférence aux responsables Erasmus des Universités et Hautes Ecoles suisses, Berne, le 17.2.02.

Nations-Unies. 1995. Guide sur la tolérance. Genève : Nations-Unies.

Office fédéral de l'éducation et de la science (OFS), ERASMUS Hochschulvertrag : Zahlen \& Fakten, Berne, 17.6.2002.

Perez S. 2002. Le concept de tolérance dans les textes internationaux in Groux, D. 2002. L'éducation à l'altérité. Paris : L'Harmattan.

Perez, S. Groux, D. \& Ferrer, F. 2001. Les relations entre l'éducation comparée et l'éducation interculturelle in Dasen, P. R. \& Perregaux, C. Eds, Pourquoi des approches interculturelles en sciences de l'éducation? Raisons éducatives, Bruxelles : De Boeck Université.

SSED. 2002. Guide de l'étudiant. Genève : SSED.

SSED. 2002. Statistiques. Genève : SSED.

Université de Genève. 2002. www.unige.ch/formev/polform/suisse.html

Union Européenne.2001. Rapport sur la Déclaration de Bologne. Bruxelles: UE.

Vincent, O. 2002. Les échanges Erasmus. Université de Genève, rectorat de Genève (groupe IRUS).

Weber, P. 1999. Communications et Coopérations interculturelles, internationales et interprofessionnelles. In Chytil, O \& Seibel F. (Eds). European Dimensions in Training and Practice of Social professions. Boskovice :Verlag ALBERT, 294303. 